Trauma Berufskrankh 2010 - 12 [Suppl 1]:9-11 DOI 10.1007/s10039-009-1554-6

Online publiziert: 29. Oktober 2009

c) Springer Medizin Verlag 2009

\author{
V. Bühren \\ BG-Unfallklinik Murnau
}

\section{Weiterentwicklung der Heilverfahren der Deutschen Gesetzlichen Unfallversicherung}

\author{
Notwendige Veränderungen aus Sicht \\ der ärztlichen Fachgesellschaften
}

Treibende Prozesse der letzten 5 Jahre für die Veränderungen in den Heilverfahren der Gesetzlichen Unfallversicherung (GUV) waren:

- die Regelungen für die ambulante Versorgung (Vertragsarztrechtveränderungsgesetz 2007),

- das Zusammengehen des Fachgebietes Orthopädie mit dem chirurgischen Schwerpunkt Unfallchirurgie in 2008 und

- die Neuordnung der gesetzlichen Unfallversicherung (Unfallversicherungsmodernisierungsgesetz 2008).

Durch die Vereinigung der gewerblichen Berufsgenossenschaften und der Unfallkassen entstand ein starker zentral ausgerichteter Verband, der unter der Bezeichnung Deutsche Gesetzliche Unfallversicherung (DGUV) firmiert. Durch die vorstehend genannten Veränderungen in der Versorgungslandschaft in Ambulanz und Klinik getriggert wird derzeit eine Diskussion geführt, wie die Steuerung des Heilverfahrens und die Zulassung von Ärzten und Krankenhäusern zukunftsweisend gestaltet werden können. Neben der DGUV sind die Fachgesellschaften der Orthopäden und Unfallchirurgen und die entsprechenden Berufs- und Interessenverbände in die Diskussion einbezogen.

\section{Zulassung zum Durchgangsarzt}

Klassischerweise ist die Facharztqualifikation Chirurgie mit einer aufbauen- den Spezialisierung in der Unfallchirurgie die Voraussetzung für die Zulassung an den Heilverfahren. Mit der Weiterbildungsordnung 1993 wurde die vorherige 6-jährige chirurgische Weiterbildungszeit auf 5 Jahre verkürzt, im Gegenzug wurde die Weiterbildungszeit im Schwerpunkt Unfallchirurgie von 2 auf 3 Jahre erweitert, was jeweils einen Gesamtzeitbedarf von 8 Jahren bedeutete. Eine wesentliche Änderung ergab sich mit der Neuordnung der chirurgischen Fächer. Das Fachgebiet Orthopädie wurde mit dem chirurgischen Schwerpunkt Unfallchirurgie vereinigt. Mit der Weiterbildungsordnung 2004 wurde der Facharzt für Orthopädie und Unfallchirurgie eingeführt, der eine Weiterbildungszeit von 6 Jahren vorsieht. Gleichzeitig wurde eine spezielle Weiterbildung „Spezielle Unfallchirurgie“ eingeführt, die aufbauend eine 3-jährige Weiterbildungszeit umfasste.

Im Vergleich zur Weiterbildungsordnung 1993 verlängerte sich somit die Regelweiterbildungszeit von 8 auf 9 Jahre. Gleichzeitig wurden die unfallchirurgischen Weiterbildungsinhalte deutlich erhöht. Mit der Weiterbildungsordnung 2004 werden 2 Jahre Basischirurgie und daran im Anschluss 4 Jahre Orthopädie und Unfallchirurgie je hälftig gefordert. Nach Absolvierung der speziellen unfallchirurgischen Weiterbildung sind somit 5 Jahre Weiterbildung in der Unfallchirurgie ergänzt um 2 Jahre in der Orthopädie absolviert. Diese Schwerpunktsetzung im unfallchirurgischen Bereich hat natur- gemäß im Gegenzug eine entsprechende Verkürzung allgemeinchirurgischer Inhalte zur Folge.

Aufgrund dieser deutlichen Ausdehnung der unfallchirurgischen Weiterbildung entstand eine Diskussion, ob tatsächlich jeder Durchgangsarzt in der Niederlassung die nunmehr verlängerte Weiterbildungszeit von mindestens 9 Jahren durchlaufen muss. Insbesondere auch der ausgiebige Operationskatalog der Speziellen Weiterbildung kann hinterfragt werden, inwieweit er für eine Tätigkeit im ambulanten Bereich nutzbringend ist. Aus dieser Überlegung entstand das Modell einer modularen Zulassung zu den Heilverfahren, die allerdings in jedem Fall die Facharztanerkennung Orthopädie und Unfallchirurgie zwingend vorschreibt. Dies bedeutet auch, dass das $\mathrm{H}$ Arzt-Verfahren (H-Arzt: Heilverfahrensarzt) auf der Grundlage anderslautender Facharztbezeichnungen entfällt.

\section{Modularisiertes D-Arzt-Verfahren}

Die Idee eines solchen beruht auf einem Basismodul mit Grundanforderungen an jeden zugelassenen Durchgangsarzt, auf dem sich weitere Module für die operative Tätigkeit, das Verletzungsartenverfahren und individuelle Zulassungen aufsetzen. Als Basisqualifikation gilt die Weiterbildung zum Facharzt für Orthopädie und Unfallchirurgie sowie zusätzlich nach der Facharztanerkennung 1 Jahr ärztliche Tätigkeit in einer Abteilung zur Behandlung 
Trauma Berufskrankh 2010 • 12 [Suppl 1]:9-11 DOI 10.1007/s10039-009-1554-6

(c) Springer Medizin Verlag 2009

\section{Bühren \\ Weiterentwicklung der Heilverfahren der Deutschen Gesetzlichen Unfallversicherung. Notwendige Veränderungen aus Sicht der ärztlichen Fachgesellschaften}

\begin{abstract}
Zusammenfassung
Tiefgreifende Veränderungen in den Rege-

lungen der ambulanten Versorgung, das Zusammengehen der Fachgebiete Orthopädie und Unfallchirurgie sowie die Neuordnung der Gesetzlichen Unfallversicherung sind Basis der Weiterentwicklung des Berufsgenossenschaftlichen Heilverfahrens. Für die Zulassung zum Durchgangsarzt (D-Arzt) sind der Abschluss der Weiterbildung zum Facharzt für Orthopädie und Unfallchirurgie als Basisqualifikation, ein weiteres Weiterbildungsjahr an einem zum Verletzungsartenverfahren (VAV) zugelassenen Haus sowie die Absolvierung berufsbegleitender Kurse für Begutachtung und Ablauf der Heilverfahren Voraussetzung. Die operative D-Arzt-Tätigkeit am Krankenhaus hat neben dem Facharzt die absolvierte Zusatzweiterbildung Spezielle Unfallchirurgie zur Bedingung. Für das VAV ist im
\end{abstract}

Anschluss an die Zusatzweiterbildung eine 3-jährige Tätigkeit an einer zum VAV zugelassenen Abteilung zu absolvieren. Für die Zulassung von Krankhäusern zum VAV ist eine Abstimmung mit dem Traumanetzwerk ${ }^{D}$ der Deutschen Gesellschaft für Unfallchirurgie vorgesehen, wobei eine Einstufung als überregionales oder regionales Traumazentrum die Basis bilden wird. Für alle Heilverfahren werden in Zukunft sowohl Bedarfsaspekte als auch qualitätssichernde Konzepte einbezogen.

\section{Schlüsselwörter}

Deutsche Gesetzliche Unfallversicherung .

Berufsgenossenschaftliches Heilverfahren .

Durchgangsarzt · Verletzungsartenverfahren . Traumanetzwerk

\section{Further developments in the medical care offered by the German statutory accident insurance. Changes needed according to the specialist medical societies}

\section{Abstract}

Far-reaching changes in the regulation of outpatient treatment, the fusion of the specialties orthopedics and trauma surgery, as well as the reorganization of statutory accident insurance form the basis for the further development of the guided care process of the German Employer's Liability Insurance Association. Certification as an authorised medical examiner requires completion of specialist training in both disciplines orthopedics and trauma surgery as the basic qualification, a further year's training in an institution certified for injury tying procedures, as well as completion of an extra-occupational course on appraisals and procedures in medical care. The position of operative medical examiner in a hospital also requires, in addition to being a specialist, completion of additional special training as trauma surgeon. For in-
\end{abstract}

jury typing procedures, 3-year's experience in a department certified in injury typing procedures is required following additional special training. Hospitals seeking certification in injury typing procedures must apply to the trauma network of the German Society of Trauma Surgery, whereby classification as a nationwide or regional trauma center forms the basis. In the future, all medical care will include requirement and quality assurance concepts.

\section{Keywords}

German statutory accident insurance. Guided care process of the German Employer's Liability Insurance Association . Authorized medical examiner · Injury typing procedures - Trauma network
Schwerunfallverletzter eines am Verletzungsartenverfahren (VAV) zugelassenen Krankenhauses, die mit einem qualifizierten Zeugnis des dort tätigen Durchgangsarztes bescheinigt werden muss.

Für eine operative Tätigkeit typischerweise als D-Arzt am Krankenhaus, gleichermaßen aber auch für niedergelassene Ärzte, die ambulante stationsersetzende operative Eingriffe umfassend durchführen wollen, gilt die Anforderung einer 3jährigen operativ ausgerichteten Zusatzweiterbildung "Spezielle Unfallchirurgie“. Dies entspricht im Übrigen der derzeit gültigen Regelung.

Für den verantwortlichen Durchgangsarzt am VAV-Krankenhaus wird nach der Zusatzweiterbildung zusätzlich und wie bisher eine 3-jährige Tätigkeit an einer zum VAV zugelassenen Abteilung im Anschluss an die Weiterbildung verlangt. Noch in der Diskussion steht die Definition eines künftigen SGB-VII-Traumazentrums (SGB: Sozialgesetzbuch), das in Anlehnung an die regionalen und überregionalen Traumazentren des Traumanetzwerk ${ }^{\mathrm{D}}$ der Deutschen Gesellschaft für Unfallchirurgie ausgerichtet werden soll. Diese Definition kann bisherige Detailregelungen, wie die 2-jährige Weiterbildungsbefugnis für die Spezielle Unfallchirurgie, überflüssig machen.

In einem dritten Modul soll die individuelle Einbeziehung spezialisierter Leistungserbringer geregelt werden. Auch für diesen Kreis wird die Erfüllung des Basismoduls Voraussetzung sein. Alternativ bleibt die Möglichkeit einer Hinzuziehung spezialisierter Ärzte durch am Verfahren beteiligte Ärzte weiterhin bestehen.

\section{Berufsbegleitende durchgangsärztliche Fortbildung}

Das Basismodul soll in Zukunft auf einem kontinuierlichen Qualitätssicherungssystem beruhen, das auch die Pflicht zu einer durchgangsärztlich spezifischen Fortbildung umfasst. Die Schwerpunkte der Fortbildung werden in den Bereichen Gutachtenwesen, Heilverfahren, Rehabilitationsmanagement und Rehabilitationsmedizin bestehen. Eingebettet werden diese Fortbildungsmaßnahmen in die schon jetzt von den Landesärztekammern geforderten Nachweise über 250 Fortbildungs- 
punkte. Ebenfalls entsprechend besteht eine Nachweispflicht im 5-jährigen Turnus, in dem im Übrigen auch eine Teilnahme an mindestens 2 unfallmedizinischen Tagungen der Landesverbände nachzuweisen ist.

Die Prozessqualität soll u. a. über die Durchgangsarztberichte im Hinblick auf Laufzeiten, Diagnostik und Diagnosestellung und die Einhaltung von Vorstellungspflichten überprüft werden. Darüber hinaus wird zu Stärkung der fachärztlichen Routine und zur Orientierung am tatsächlichen Bedarf eine Erhöhung der heute geltenden Mindestfallzahl von 150 Fällen diskutiert.

\section{Eckpunkte eines künftigen Verletzungsartenverfahrens}

In Analogie zum Traumanetzwerk der DGU sind synergistisch SGB-VIITraumanetzwerke angedacht, in die komplizierte und folgenträchtige Verletzungsfälle gesteuert werden sollen. Ziele sind eine Kooperation der beteiligten Leistungsbereiche und insbesondere eine strukturierte Zusammenarbeit im Sinne der Frührehabilitation schwerer und schwerster Verletzungen. Auch für diesen Bereich sind eine Qualitäts- und Bedarfssteuerung, z. B. über Mindestfallzahlen, angedacht, die in der Größenordnung von 100 VAV-Fällen pro Jahr liegen könnten.

\section{Fazit}

Unter dem Druck sozialpolitischer Entwicklungen und den Veränderungen in der medizinischen Fachgebiets- und Versorgungslandschaft ist eine Diskussion zur Ausgestaltung zukünftiger D-Arztund VAV-Verfahren entstanden. Im ambulanten Bereich zeichnet sich die Einführung eines modularen D-Arzt-Verfahrens ab, das das bisherige H-Arzt-Verfahren überflüssig machen wird. Bedarfsgesichtspunkte und Qualitätssicherungen werden in die Prozedur integriert. Die Diskussionen für die Ausprägung der stationären Verfahren und insbesondere des VAV laufen derzeit noch in enger Abstimmung mit den Fachgesellschaften. Auch hier sind eine stärkere Bedarfs- und Qualitätsorientierung absehbar.

\section{Korrespondenzadresse}

Prof. Dr. V. Bühren

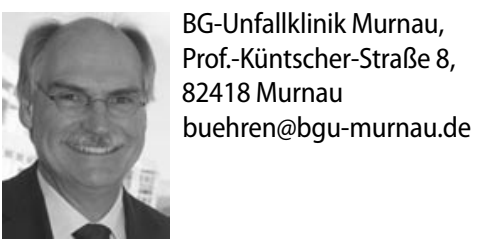

Interessenkonflikt. Der korrespondierende Autor gibt an, dass kein Interessenkonflikt besteht. 